\title{
Effect of empagliflozin on ectopic fat stores and myocardial energetics in type 2 diabetes: the EMPACEF study
}

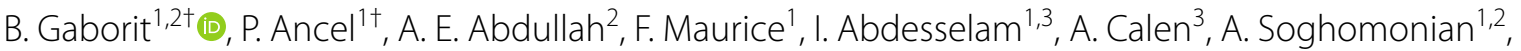 \\ M. Houssays ${ }^{4}$, I. Varlet ${ }^{3}$, M. Eisinger ${ }^{1,2}$, A. Lasbleiz ${ }^{1,3}$, F. Peiretti ${ }^{1}$, C. E. Bornet ${ }^{5}$, Y. Lefur ${ }^{3}$, L. Pini ${ }^{3}$, S. Rapacchi ${ }^{3}$, \\ M. Bernard ${ }^{3}$, N. Resseguier ${ }^{5,6}$, P. Darmon ${ }^{1,2}$, F. Kober $^{3 \dagger}$ and A. Dutour ${ }^{1,2^{*}+}$
}

\begin{abstract}
Background: Empagliflozin is a sodium-glucose cotransporter 2 (SGLT2) inhibitor that has demonstrated cardiovascular and renal protection in patients with type 2 diabetes (T2D). We hypothesized that empaglifozin (EMPA) could modulate ectopic fat stores and myocardial energetics in high-fat-high-sucrose (HFHS) diet mice and in type 2 diabetics (T2D).

Methods: C57BL/6 HFHS mice $(n=24)$ and T2D subjects $(n=56)$ were randomly assigned to 12 weeks of treatment with EMPA (30 mg/kg in mice, $10 \mathrm{mg} /$ day in humans) or with placebo. A $4.7 \mathrm{~T}$ or $3 \mathrm{~T} \mathrm{MRI}$ with ${ }^{1} \mathrm{H}-\mathrm{MRS}$ evaluationmyocardial fat (primary endpoint) and liver fat content (LFC)-were performed at baseline and at 12 weeks. In humans, standard cardiac MRI was coupled with myocardial energetics (PCr/ATP) measured with ${ }^{31}$ P-MRS. Subcutaneous (SAT) abdominal, visceral (VAT), epicardial and pancreatic fat were also evaluated.

The primary efficacy endpoint was the change in epicardial fat volume between EMPA and placebo from baseline to 12 weeks. Secondary endpoints were the differences in PCr/ATP ratio, myocardial, liver and pancreatic fat content, SAT and VAT between groups at 12 weeks.

Results: In mice fed HFHS, EMPA significantly improved glucose tolerance and increased blood ketone bodies (KB) and $\beta$-hydroxybutyrate levels $(p<0.05)$ compared to placebo. Mice fed HFHS had increased myocardial and liver fat content compared to standard diet mice. EMPA significantly attenuated liver fat content by 55\%, $(p<0.001)$ but had no effect on myocardial fat.

In the human study, all the 56 patients had normal LV function with mean LVEF $=63.4 \pm 7.9 \%$. Compared to placebo, T2D patients treated with EMPA significantly lost weight (-2.6 $\mathrm{kg}[-1.2 ;-3.7])$ and improved their HbA1c by $0.88 \pm 0.74 \%$. Hematocrit and EPO levels were significantly increased in the EMPA group compared to placebo $(p<0.0001, p=0.041)$. EMPA significantly increased glycosuria and plasma KB levels compared to placebo $(p<0.0001$, $p=0.012$, respectively), and significantly reduced liver fat content ( $-27 \pm 23 \mathrm{vs} .-2 \pm 24 \%, p=0.0005)$ and visceral fat $(-7.8 \%[-15.3 ;-5.6]$ vs. $-0.1 \%[-1.1 ; 6.5], p=0.043)$, but had no effect on myocardial or epicardial fat. At 12 weeks, no significant change was observed in the myocardial PCr/ATP ( $p=0.57$ between groups).
\end{abstract}

*Correspondence: anne.dutour@ap-hm.fr

${ }^{\dagger}$ B. Gaborit, P. Ancel, F. Kober, A. Dutour contributed equally to the work

${ }^{1}$ Aix Marseille Univ, INSERM, INRAE, C2VN Marseille, France

Full list of author information is available at the end of the article

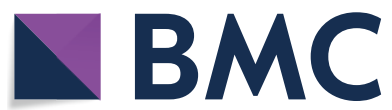

c The Author(s) 2021. This article is licensed under a Creative Commons Attribution 4.0 International License, which permits use, sharing, adaptation, distribution and reproduction in any medium or format, as long as you give appropriate credit to the original author(s) and the source, provide a link to the Creative Commons licence, and indicate if changes were made. The images or other third party material in this article are included in the article's Creative Commons licence, unless indicated otherwise in a credit line to the material. If material is not included in the article's Creative Commons licence and your intended use is not permitted by statutory regulation or exceeds the permitted use, you will need to obtain permission directly from the copyright holder. To view a copy of this licence, visit http://creativeco mmons.org/licenses/by/4.0/. The Creative Commons Public Domain Dedication waiver (http://creativecommons.org/publicdomain/ zero/1.0/) applies to the data made available in this article, unless otherwise stated in a credit line to the data. 
Conclusions: EMPA effectively reduced liver fat in mice and humans without changing epicardial, myocardial fat or myocardial energetics, rebutting the thrifty substrate hypothesis for cardiovascular protection of SGLT2 inhibitors.

Trial registration NCT, NCT03118336. Registered 18 April 2017, https://clinicaltrials.gov/ct2/show/NCT03118336 Keywords: SGLT2 inhibitors, Epicardial adipose tissue, Ectopic fat, Myocardial energetics, Pcr/atp, ${ }^{31}$ P-MRS, MRI

\section{Background}

Sodium-glucose cotransporter 2 (SGLT2) inhibitors are a unique class of oral antidiabetic medications that reduce glucose reabsorption in the renal proximal tubules, thereby enhancing urinary glucose excretion. Large randomized controlled trials on the SGLT2 inhibitors, empagliflozin (EMPA-REG OUTCOME), canagliflozin (CANVAS PROGRAM), and dapagliflozin (DECLARE-TIMI 58) have been found to markedly reduce cardiovascular events and heart failure hospitalizations and to significantly improve renal outcomes in patients with type 2 diabetes (T2D) with a high or very high cardiovascular risk [1-3]. Multiple hypotheses have been proposed to explain the beneficial effects of SGLT2 inhibitors, which could be multifactorial, the most common one being the effect on diuresis/natriuresis [4]. In a hypertensive heart failure rat model, empagliflozin improved hemodynamics, attenuated intra-cardiac fibrosis and modulated genes related to fatty acid metabolism (PPAR and ACADM) [5]. In LPS-treated cardiomyocytes and macrophages and in LPS-treated mice, empagliflozin reduced inflammation, promoted the expression of anti-inflammatory M2 marker proteins, and activated AMPK, preventing ATP/ADP depletion [6]. In an experimental metabolic syndrome with heart failure with preserved ejection fraction (HFpEF) in the ZFS1 rat, empagliflozin improved endothelial function, cardiac remodeling, and senescence, and reduced perirenal fat [7]. However, the mechanisms involved in these early impressive cardiac benefits are still not completely understood. Type 2 diabetes is associated with an increase in ectopic fat deposition in the heart, liver and pancreas. One anatomic supplier of substrates to the myocardium is cardiac ectopic fat. No fascia separates epicardial adipose tissue adipocytes from cardiomyocytes and the coronary blood stream. Myocardial triglyceride content has been suggested to be an energy reserve for the myocardium in contexts of high energy demand [8]. Previous studies did not observe any significant association between the phosphocreatine (PCr)/ adenosine triphosphate ratio, a recognized in vivo marker of myocardial energy metabolism and cardiac fat compartments [9]. We previously demonstrated that patients with T2D have increased epicardial and myocardial fat and that these ectopic fat stores could negatively affect left ventricular function and contribute to coronary artery disease (CAD) or heart failure $[10,11]$. Here we examined whether SGLT2 inhibitors had an impact on ectopic fat stores and myocardial energetics in humans and mice. Impairment in myocardial high-energy phosphate metabolism and bioenergetics, characterized by a reduced cardiac phosphocreatine-to-ATP ratio (PCr/ATP), has been postulated to play a major role in the development and progression of heart failure $[12,13]$. Adenosine triphosphate (ATP) is essential for normal cardiac function, including myofibrillar contraction, ion transport, and myocyte viability. Also, the $\mathrm{PCr} / \mathrm{ATP}$ ratio, which is a powerful index of the energetic state of the heart [14] correlates with heart failure severity and is a strong predictor of cardiovascular mortality [15]. In the diabetic heart developing heart failure, metabolic flexibility of the heart allows oxidation of other substrates such as ketone bodies (KB), lactate and branched-chain amino acids. Interestingly, KB are more energetically efficient than other substrates, requiring the lowest oxygen consumption $[16,17]$. Empagliflozin increases plasma ketone body levels, and it has therefore been hypothesized that a shift in energy substrate metabolism towards KB or an increased availability of ketones explains the positive cardiovascular outcomes in the EMPA-REG OUTCOME study. A recent study using ${ }^{11} \mathrm{C}$-palmitate and ${ }^{18} \mathrm{~F}$-FDG PET/CT reported no change in myocardial FFA uptake but a significant $57 \%$ reduction in myocardial glucose uptake in T2D individuals treated with empagliflozin [18].

Multiple imaging modalities such as ${ }^{31} \mathrm{P}$-MRS and ${ }^{1} \mathrm{H}$ MRS allow cardiac energetics and ectopic fat stores to be measured in vivo noninvasively [19]. We investigated the effect of 12 weeks of empagliflozin treatment on ectopic fat stores (except for epicardial fat) in mice fed a high-fat highsucrose diet (HFHS) and on ectopic fat stores and myocardial energetics ( $\mathrm{PCr} / \mathrm{ATP})$ in T2D patients.

In the human study, the primary efficacy endpoint was the change in epicardial fat volume between EMPA and placebo from baseline to 12 weeks. Secondary end points were the differences in PCr/ATP ratio, myocardial, liver and pancreatic fat content, SAT and VAT between groups at 12 weeks.

\section{Methods}

\section{Mouse study}

Animals and experimental procedure

All the animal procedures were approved by the ethics committee of Aix-Marseille University and complied with the European Convention for the protection of animals used for experimental purposes. Animal MRI 
experiments were carried out on a 4.7T Bruker Biospec Avance system (Bruker, Ettlingen, Germany) dedicated to rodent exploration and equipped with a $70 \mathrm{~mm}$ birdcage TX resonator and a decoupled surface receive coil.

Thirty-four C57BL/6 8-week-old male mice (Janvier, France) were housed in a controlled environment under standard laboratory conditions (12-12 h light-dark cycle, $22^{\circ} \mathrm{C}$ ). The mice were randomly divided into: Control $(n=10)$ with standard diet (\#U8224G10R, Safe Diets, France) and HFHS $(n=24)$ diet (\#U8978P Version 0019, Safe Diets, France) as previously described [20] (Fig. 1). At 4 weeks, metabolic status (intraperitoneal glucose tolerance test (IPGTT), glucagon, insulin and blood ketones) and MRI evaluation of ectopic fat stores (liver and myocardial, as rodents have no epicardial fat) were performed. The HFHS group was then randomly divided into two subgroups, EMPA $(n=12)$, with empagliflozin in water $(30 \mathrm{mg} / \mathrm{kg}$ bodyweight $+0.1 \%$ DMSO (Sigma, USA)), and a placebo group HFHS $(n=12)$ with $0.1 \%$ DMSO in water. Metabolic status and MRI evaluation were performed after 4 and 12 weeks of treatment. At 24 weeks, mice were sacrificed, and tissues (heart, liver) collected (Additional file 1: Appendix S1).

\section{Human study}

\section{Study design}

This randomized, parallel-group, double-blind, phase 3 trial was performed at the Endocrinology, Metabolic Diseases and Nutrition Department (Marseille, France) with a 1:1 allocation to treatment arms. The local ethics committee (CPP Ile-de-France VII/ANSM) approved all the trial procedures in compliance with the Declaration of Helsinki. The EMPACEF clinical trial was registered at clinicaltrials.gov (NCT03118336).

Patients Subjects diagnosed with T2D, on stable antidiabetic medication, were recruited and randomly assigned to empagliflozin (10 mg/day) or placebo treatment. Before inclusion, all the patients gave their written informed consent.

Principal inclusion criteria were T2D (as defined by the World Health Organization criteria [21]), age $\geq 18$ years, HbA1c 7-10\%, renal function (MDRD) $>60 \mathrm{~mL} / \mathrm{min}$, on glucose-lowering therapy (metformin, sulfonylureas, glinides, dipeptidyl peptidase-4 (DPP-4) inhibitors, GLP-1 receptor agonists or basal insulin) at a stable dose for at least 3 months.

Participants were excluded if they had uncontrolled hyperglycemia at screening (fasting blood glucose $\mathrm{FBG} \geq 240 \mathrm{mg} / \mathrm{dL}$, were pregnant/breastfeeding or treated with ongoing corticoids or thiazolidinedione, had acute coronary syndrome or unstable angina during the last 3 months, or stage 4 lower limb peripheral artery disease (PAD) or previous history of lower limb amputations, any contraindication to magnetic resonance imaging, or more than $5 \%$ total body weight loss within the last month.

Randomization and masking Participants were randomly assigned by a stratified computed randomization procedure accounting for sex, age, and BMI to the empagliflozin group (EMPA) or placebo and were masked to the treatment assignment. Randomization and assignment to the double-blind trial was done by the central pharmacist. Blinding of investigators and patients was ensured by supplying EMPA and placebo tablets with identical appearance and packaging. Unblinding was performed by blinded research assistants after all the MRI post-processing measurements had been made.

Procedures Patients received one individual dietary counseling session before the baseline visit, according to American Diabetes Association recommendations [22]. Patient education, clinical examination with anthropometrics (height, weight, BMI, waist circumference), systolic and diastolic blood pressure blood sampling (FBG, lipid profile, uricemia, hematocrit, erythropoietin, ketonemia, glycosuria, PAI-1, ferritin, liver enzymes) treatment safety, and adherence were assessed at weeks 0 (Visit 1 V1), 4 (V2) and 12 (V3).

Enrolled patients were allocated to one treatment arm, empagliflozin $10 \mathrm{mg}$ once daily or matching placebo orally (Boehringer Ingelheim, Ingelheim/Rhein, Germany) for 12 weeks.

Magnetic resonance imaging All human MR imaging and spectroscopy was carried out on a $70 \mathrm{~cm}$ diameter 3 T MR system (Verio, Siemens Healthineers, Erlangen, Germany) equipped with a 32-element phased array coil. Visceral abdominal adipose tissue area was measured with a $5 \mathrm{~mm}$ transverse slice at L4-L5 intervertebral disk level, using a 3-point Dixon sequence at baseline (week 0) and 12 weeks, as previously described [23].

Liver fat, pancreatic fat and myocardial triglyceride content were quantified with ${ }^{1} \mathrm{H}$-spectroscopy, using a single voxel PRESS sequence, as previously described $[11,24]$. Epicardial fat volume and cardiac function were assessed, as previously described [10], using a multislice steady-state free precession cine sequence in short-axis view that covered the LV from base to apex and were manually contoured (epicardial and endocardial left ventricular contours) using dedicated post-processing software (Argus, Siemens Medical Solutions). Left ventricular ejection fraction (LVEF) and LV mass were 


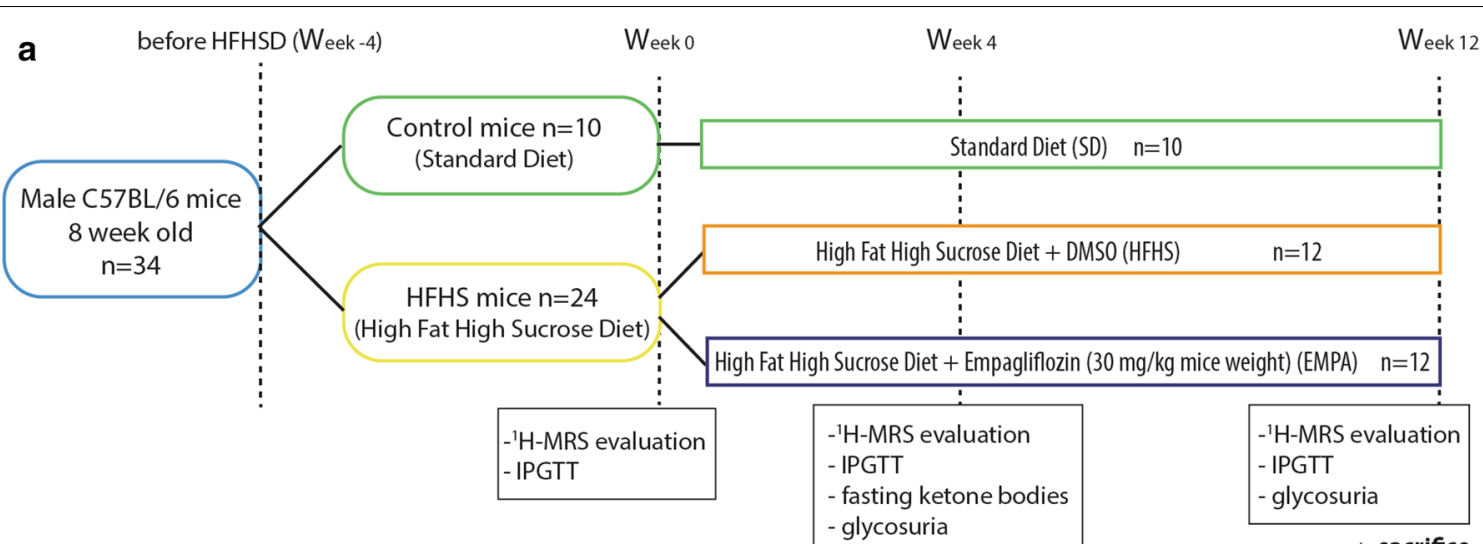

b
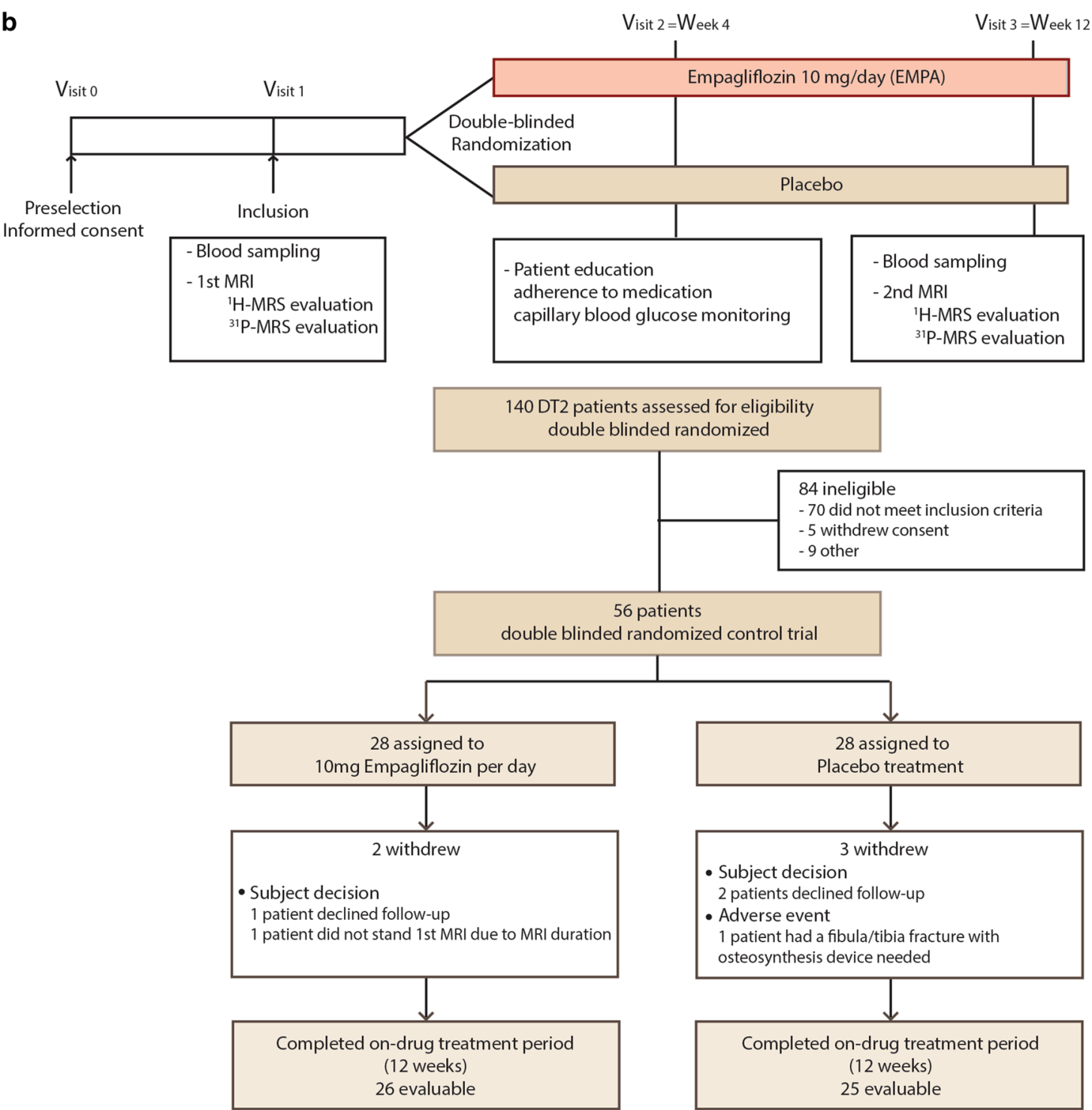

Fig. 1 Study design and flow chart. a Mice design. b Human design. IPGTT, intraperitoneal glucose tolerance test; MRI, magnetic resonance imaging; MRS, magnetic resonance spectroscopy 
calculated with Argus software. LV mass was indexed with body surface area, as previously described [10].

${ }^{31} \mathrm{P}$ nuclear magnetic resonance spectroscopy A ${ }^{31} \mathrm{P}$ Surface Heart Coil (Rapid Biomedical GmbH, Rimpar, Germany, O-XQ-030-01,605 V01) with a working frequency of $49.9 \mathrm{~Hz}$ was used for radiofrequency transmit and receive. A $3 \mathrm{D}$ chemical shift imaging (CSI) sequence was used to acquire phosphorus MR spectroscopic imaging data (TR 1300 ms, flip angle 70 degrees, Hamming acquisition $k$-space weighting with six excitations in the center of $k$-space). The sequence was run without ECG or respiratory gating. The radiofrequency voltage necessary to produce a flip angle of 90 degrees in the centrally placed PPA tube (10 mL of $0.6 \mathrm{M}$ phenyl phosphoric acid) on the surface coil was determined beforehand using a calibration scan. Spectroscopic acquisition time was $34 \mathrm{~min}$ owing to a relatively small voxel size $(15 \times 15 \times 24 \mathrm{~mm})$.

${ }^{31} \mathrm{P}$ spectra were analyzed according to Bottomley [25] using software developed in-house [26] running in the IDL framework (Interactive Data Language; ITT Visual Solutions, Boulder, CO, USA). Voxels were placed in the mid inter-ventricular septal wall across a stack of shortaxis images, starting from the base of the heart in a slice where left and right ventricles were visually comparable in terms of surface area (approximately at LV mid-level).

A ${ }^{31} \mathrm{P}$ SMR spectrum was obtained from each voxel after spectral filtering at $15 \mathrm{~Hz}$ and adjusting both phase and frequency shift to obtain a $\mathrm{PCr}$ peak at $0 \mathrm{ppm}$. Spectra were later fitted using AMARES (Advanced Method for Accurate, Robust, Efficient Spectral Fitting) [27]. The ratio was corrected for the contribution of ATP in the left ventricular blood to the myocardial ATP resonance [25]. A previously built $\mathrm{B}^{+}$map of the surface coil was used to estimate the local flip angle at the location of each voxel. Signal amplitudes were then corrected for partial saturation using this estimated local flip angle and literature T1 values of the corresponding resonances [28]. The ratio of the corrected $\mathrm{PCr}$ to $\gamma$-ATP signal amplitudes was used to generate a PCr/ATP concentration ratio $[29,30]$.

Human study outcomes The primary efficacy endpoint was defined as the difference in change in epicardial fat volume between EMPA and placebo from baseline to 12 weeks of treatment. Secondary endpoints were the differences in changes in PCr/ATP ratio, myocardial, liver and pancreatic fat content, subcutaneous and visceral abdominal fat between EMPA and placebo from baseline to 12 weeks.

Power calculation The sample size calculation was based on our previous work on exenatide treatment and ectopic fat stores [24]. Sample size calculation was performed under the following hypotheses: (i) a difference between EMPA group and placebo group regarding the primary endpoint of 7.2, (ii) a standard deviation for both groups regarding the primary endpoint of 7.7, (iii) an allocation ratio of $1: 1$, (iv) a power of 0.8 and a bilateral alpha risk of 0.05 . A total of 38 patients were required (19 in each group). Allowing for a risk of loss to follow-up of $10 \%$ and technical MRI issues, the sample size was increased to 56.

\section{Statistical analyses}

Statistical analyses were performed using Prism v8 (Graphpad, San Diego, CA, USA) or SPSS v23 (Chicago, USA) and the significance threshold was $p=0.05$. Results are presented as means \pm standard deviation or median (25th, 75 th percentile). Normality of variables was analyzed by the Shapiro-Wilk normality test. Datasets were compared using paired Student's $t$-tests for normal distribution, with either a Mann-Whitney $U$-test or Wilcoxon signed rank test if not parametric. Two-way repeated measures ANOVA was performed with treatment as factor to test time and group interaction. Intention-to-treat analysis was performed for primary outcome assessment using the Last Observation Carried Forward (LOCF) method. Per-protocol analysis was then performed.

\section{Results}

\section{Mouse study}

Mice fed HFHS became obese and glucose-intolerant at 4 weeks compared to wild-type mice fed standard diet (Fig. 2). As expected, empagliflozin treatment significantly reduced weight gain at 4 weeks $(p<0.001)$, increased daily water intake $(p<0.001)$, blood ketone bodies and $\beta$-hydroxybutyrate levels $(p<0.05)$ and glycosuria $(p<0.001)$ compared to HFHS mice fed placebo. EMPA treatment significantly improved glucose tolerance in the iPGTT test at 4 and 12 weeks, and decreased insulin/glucagon basal ratio at 12 weeks compared to HFHS mice $(p<0.001)$ (Fig. 2).

Mice fed HFHS had increased myocardial and liver fat content measured in vivo with ${ }^{1} \mathrm{H}$-MRS at 4 weeks compared to wild-type mice fed standard diet (Fig. 2). EMPA treatment significantly attenuated liver fat content (LFC) by $55 \%$, compared to HFHS mice: LFC: $35.2 \pm 10.3 \%$ EMPA vs. $77.9 \pm 6.5$ HFHS, $p<0.001)$ but had no effect on myocardial fat $(p=\mathrm{NS})$. After sacrifice, oil-red-O staining showed a protective effect of EMPA treatment on liver neutral lipid content, but no effect on myocardial triglyceride accumulation compared to HFHS mice.

Of note, mice fed HFHS displayed a reduced oxidative capacity, as expressed by a significant decrease in the enzymes involved in the KB metabolism bdh1, bdh2, hmgcs 2 and oxct1 mRNA levels, compared to wild-type 

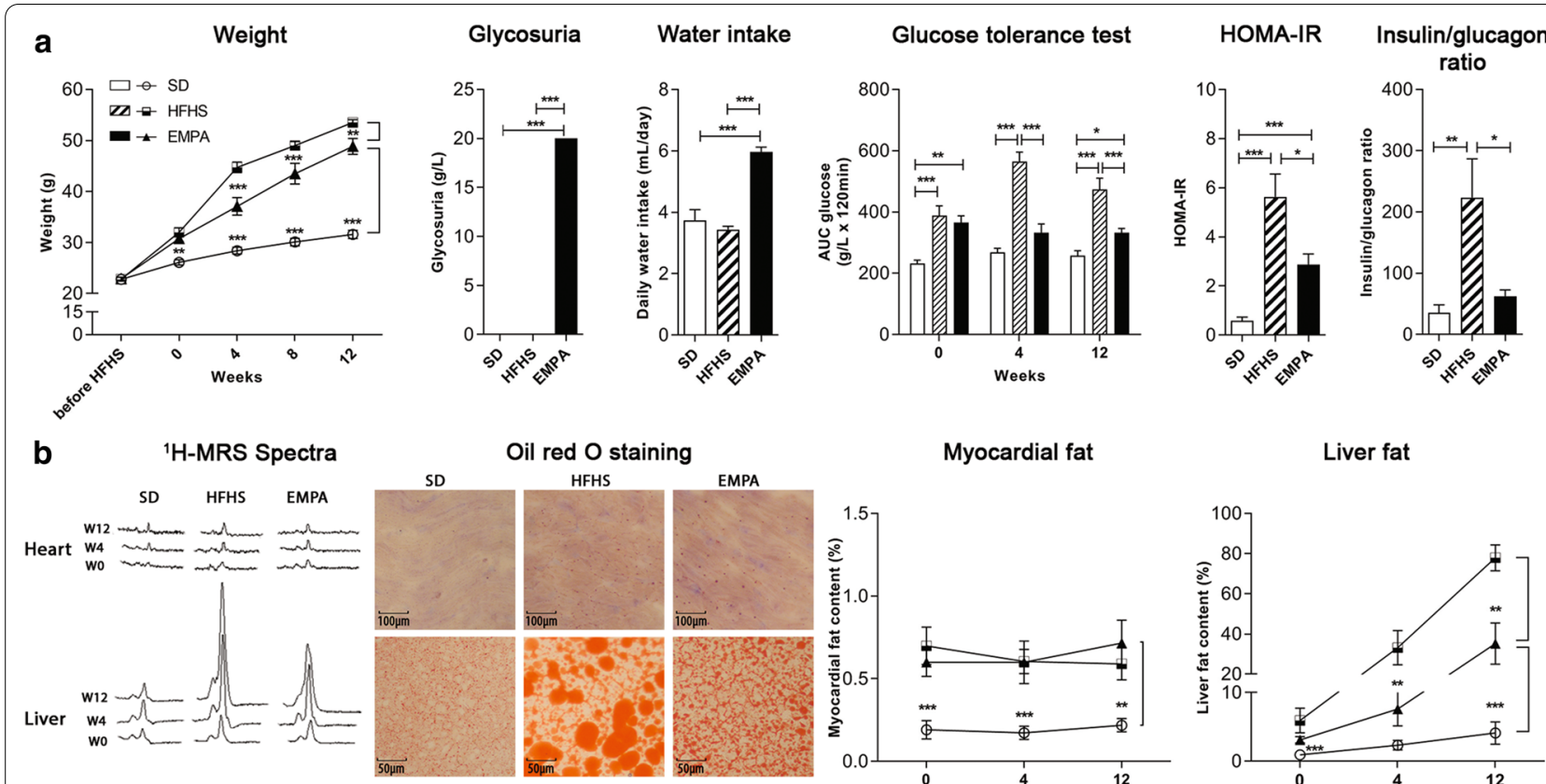

Oil red $\mathrm{O}$ staining
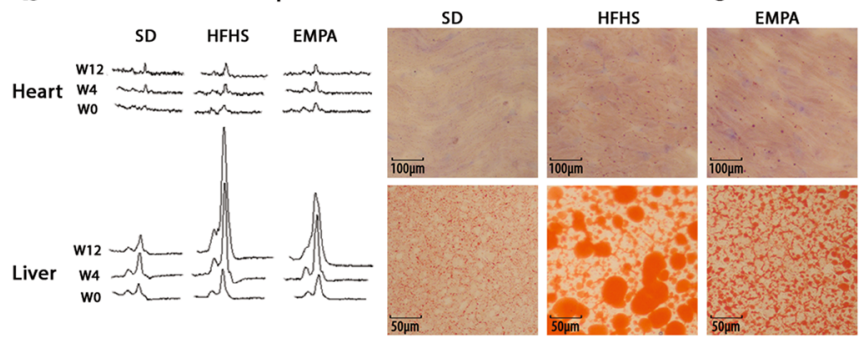

Myocardial fat

\section{Liver fat}
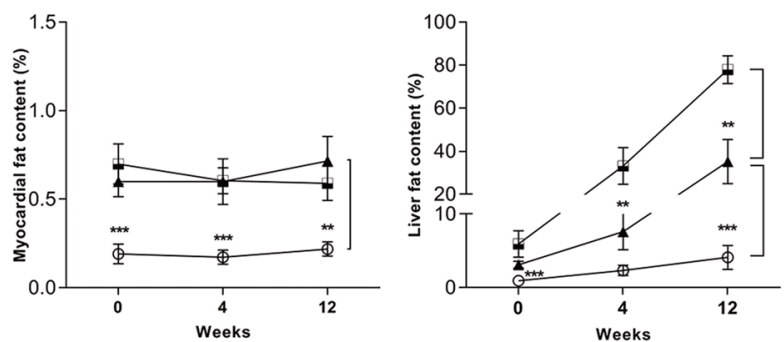

C

Ketone bodies

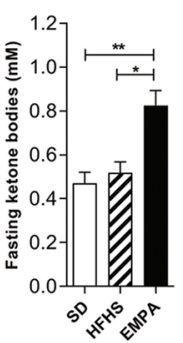

$\beta$-hydroxybutyrate

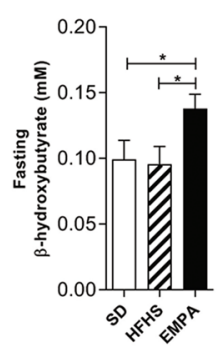

Bdh1

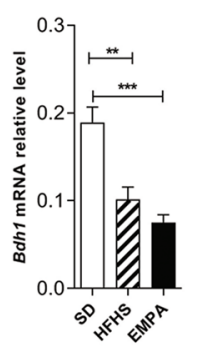

Bdh2

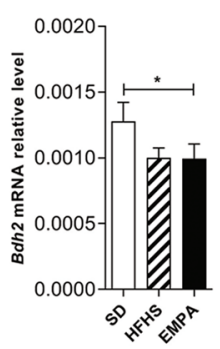

Hmgcs2

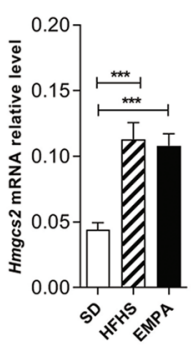

Oxct1

Fig. 2 Changes in metabolic profile and ectopic fat stores at 12 weeks of treatment in mice. a Metabolic profile: Weight of mice during 12 weeks of standard diet (SD), High Fat High Sucrose diet (HFHS) or HFHS + empagliflozin (EMPA); glycosuria at 4 and 12 weeks in SD, HFHS and EMPA mice; mean daily water intake ( $\mathrm{mL} /$ day) in SD, HFHS and EMPA mice measured every 2 days for 12 weeks; intraperitoneal glucose tolerance test (IPGTT) in SD, HFHS and EMPA mice measured at week 0, 4 and 12 of empagliflozin treatment; HOMA-IR index and insulin/glucagon ratio of SD, HFHS and EMPA mice measured at 12 weeks. b Ectopic fat stores: Evolution of typical ${ }^{1} \mathrm{H}$-MRS spectra (triglycerides peak at 1.3 ppm) acquired in the heart or liver of SD, HFHS and EMPA mice at week 0,4 and 12; Representative Oil-Red-O stain images ( $n=10$ images per mouse) of lipid droplets in heart and liver from SD, HFHS or EMPA mice; scale bars, $100 \mu \mathrm{m}$ for heart and $50 \mu \mathrm{m}$ for liver. $\mathbf{c}$ Ketone pathways: Ketone bodies and fasting $\beta$-hydroxybutyrate of SD, HFHS and EMPA mice measured at 4 weeks; RT-qPCR analyses of genes coding for enzymes involved in $\beta$-oxidation pathway (Bdh1, Bdh2, Hmgcs2, Oxct1) of SD, HFHS and EMPA mice after 12 weeks. SD $(n=10)$, HFHS $(n=12)$, EMPA $(n=12)$. Data represent mean values \pm SEM. Differences between SD, HFHS and EMPA were determined by nonparametric unpaired Mann-Whitney comparative tests or ANOVA with Tukey's post hoc test; ${ }^{*} p<0.05,{ }^{* *} p<0.01,{ }^{* *} p<0.001$ (from post hoc test)

mice $(p<0.05)$. Empagliflozin treatment did not modify the expression of these genes compared to HFHS mice (Fig. 2).

\section{Human study}

Of the 140 T2D patients screened for the study, 84 were ineligible (Fig. 1) and 56 were included and randomly assigned to EMPA $(n=28)$ or placebo $(n=28)$, and received at least one dose of the study medication. Of all the randomized patients, only 51 completed the trial, (owing especially to MRI issues), and had complete ${ }^{1} \mathrm{H}$ MRS (Fig. 1). Owing to technical issues, only 34 patients (18 in the EMPA and 16 in the placebo group) had the two ${ }^{31} \mathrm{P}$-MRS evaluations. In the placebo group, there was one major adverse event declared with an accidental fibula/tibia fracture that needed osteosynthesis and contraindicated the patient for the second MRI. 


\section{Patient characteristics}

The mean age of the study population was $56.9 \pm 9.6$ years, with mean BMI $34.9 \pm 6.0 \mathrm{~kg} / \mathrm{m}^{2}, \mathrm{HbA} 1 \mathrm{c}$ $8.13 \pm 1.05 \%$ and mean disease duration $11.1 \pm 6.7$ years. Baseline anthropometric and clinical characteristics were all comparable between EMPA and placebo (Table 1). Physical activity did not differ between groups either at baseline or from baseline to 12 weeks.

\section{Intention-to-treat analysis}

\section{Effect of EMPA on body composition and metabolic parameters}

EMPA resulted in a significant weight loss of $-2.6 \mathrm{~kg}$ $[-3.7 ;-1.2]$ at V3 $(\mathrm{p}<0.0001)$ (Table 2). EMPA significantly improved glycemic control with a significant decrease in fasting blood glucose $(-2.24 \pm 2.30 \mathrm{mmol}$ $(p<0.0001$ for time) and HbA1c by $0.88 \pm 0.74 \%$ ( $p<0.0001$ time). Compared to placebo, these improvements were significant $(p=0.006$, and $p=0.003$ respectively). A significant decrease in uric acid was observed in the EMPA group compared to the placebo group $(p=0.005)$.

\section{Effect of EMPA on epicardial fat volume}

All the patients had normal LV function with mean LVEF $=63.5 \pm 8.2 \%$. We observed no significant change in epicardial fat volume from baseline to 12 weeks in the EMPA group (V1 $108.5 \pm 31.8$ vs. V3 $106.9 \pm 31.8 \mathrm{~mL}$ $p=0.09$ ) and in the placebo group (V1 $105.8 \pm 21.3$ vs. $105.3 \pm 20.7 \mathrm{~mL}$ at V3, $p=0.34)(p=0.88$ for the difference between groups). No change in LV mass, LVMi, or LVEF was found at 12 weeks between groups (Table 2).

Table 1 Baseline characteristics of the study population at baseline

\begin{tabular}{lllr}
\hline & Placebo $(\boldsymbol{n}=\mathbf{2 5})$ & Empa $(\boldsymbol{n = 2 6 )}$ & $\boldsymbol{p}$ \\
\hline Age (years) & $58.6 \pm 9.2$ & $57.0 \pm 10.1$ & 0.483 \\
Sex ratio $(\mathrm{M}, \%)$ & $10(40)$ & $10(38)$ & $>0.999$ \\
BMI $\left(\mathrm{kg} / \mathrm{m}^{2}\right)$ & $34.1[30.5 ; 39.5]$ & $32.6[31.8 ; 36.6]$ & 0.644 \\
Diabetes duration (years) & $9[6 ; 18]$ & $9.5[7 ; 16.3]$ & 0.862 \\
HbA1c (\%) & $8.06 \pm 1.10$ & $8.20 \pm 1.01$ & 0.653 \\
Hypertension $(n, \%)$ & $13(52)$ & $19(73)$ & 0.153 \\
Dyslipidemia $(n, \%)$ & $19(76)$ & $18(69)$ & 0.755 \\
Smoking status active & $5(20)$ & $5(19)$ & $>0.999$ \\
$\quad(n, \%)$ & & & \\
History of CAD/PAD/stroke & $7(28)$ & $9(35)$ & 0.765 \\
Very high risk $(n, \%)^{a}$ & $19(76 \%)$ & $20(77 \%)$ & $>0.999$ \\
\hline
\end{tabular}

BMI: body mass index; CAD: coronary artery disease; PAD: peripheral artery disease; ESC: European Society of Cardiology

a Cardiovascular risk according to European Society of Cardiology guidelines[60]

\section{Effect of EMPA on myocardial energetics}

As expected, EMPA treatment significantly increased glycosuria and $\mathrm{KB}$ levels (Fig. 3a) compared to placebo $(p<0.0001, p=0.01$, respectively). At 12 weeks, no significant change was observed in the $\mathrm{PCr} / \mathrm{ATP}$ ratio either in the EMPA, or in the placebo group (Fig. 3a). Of note, hematocrit and EPO were significantly increased in the EMPA group compared to placebo $(p=0.0001$ and $p=0.04$, respectively) (Table 2 ).

\section{Effect of EMPA on other ectopic fat stores}

All the patients except for two in the placebo group had NAFLD (LFC > 5\%) at baseline. LFC was comparable between groups $(p=0.25)$ at baseline and markedly decreased in the EMPA group by $27 \%$ from 22.5 [12.641.1 ] to 19.9 [8.49-28.3], compared to placebo by $2 \%$ from 31.5 [18.5-54.9] to 29.6 [16.7-55.7] $p<0.0001$ for time, and $p=0.0005$ for the difference between groups (Fig. 3b). This decrease was accompanied by a significant decrease in liver enzymes ALT and GGT in the EMPA group compared to placebo (for time $p=0.03$, $p=0.0002$ respectively, but this did not reach significance for the difference between groups, $p=0.07$ and $p=0.22$ respectively) (Table 1 ). Weight loss $\geq 5 \%$ occurred in $38 \%$ of patients on EMPA and in $12 \%$ of patients on placebo $(p=0.052)$. The LFC change was significantly correlated with weight loss $(r=0.39, p=0.006)$. Patients in the EMPA group exhibited significant reduction of visceral abdominal fat $\left(211.2 \pm 31.4\right.$ at V1 to $199.1 \pm 38.7 \mathrm{~cm}^{2}$ at $\mathrm{V} 3, p=0.04)$, compared to patients in the placebo group $\left(228.7 \pm 74.1\right.$ at $\mathrm{V} 1$ to $232.3 \pm 71.6 \mathrm{~cm}^{2}$ at V3, $\left.p=0.46\right)$, $p=0.04$ between groups (Fig. 3c). Interestingly, no significant difference between groups was observed for subcutaneous fat, myocardial fat or pancreatic fat (Fig. 3b, c).

\section{Per-protocol analysis}

Per-protocol analysis confirmed the results obtained in ITT analysis with no significant reduction of EAT, myocardial, or pancreatic fat, but a significant reduction of visceral fat and LFC (EMPA $n=25$ and placebo $n=23$ per group $p=0.04$ and $p=0.0011$, respectively).

\section{Discussion}

This trial provides evidence that empagliflozin effectively reduces liver steatosis in mice and humans compared with placebo but has no immediate effects on cardiac ectopic fat or myocardial energetics. Interestingly, these effects occurred as early as 12 weeks after treatment initiation in the presence of moderate weight loss and significant improvement in glycemic profile. 
Table 2 Baseline characteristics and changes in parameters after 12 weeks of treatment

\begin{tabular}{|c|c|c|c|c|c|c|c|}
\hline & \multicolumn{3}{|l|}{ Placebo $(n=25)$} & \multicolumn{3}{|l|}{ Empa $(n=26)$} & \multirow{2}{*}{$\begin{array}{l}\text { Difference } \\
\text { between groups } \\
p\end{array}$} \\
\hline & Baseline & Posttreatment & $p$ & Baseline & Posttreatment & $p$ & \\
\hline \multicolumn{8}{|l|}{ Clinical parameters } \\
\hline Systolic blood pressure $(\mathrm{mm} \mathrm{Hg})$ & $124 \pm 16$ & $124 \pm 13$ & 0.9395 & $125 \pm 9$ & $124 \pm 9$ & 0.5717 & 0.7109 \\
\hline Diastolic blood pressure $(\mathrm{mm} \mathrm{Hg})$ & $74 \pm 11$ & $73 \pm 10$ & 0.8425 & $71 \pm 8$ & $71 \pm 10$ & 0.9717 & 0.9063 \\
\hline Weight $(\mathrm{kg})$ & $93[83 ; 105]$ & $94[82 ; 104]$ & 0.8882 & $91[81 ; 105]$ & $89[80 ; 102]$ & $<0.0001$ & 0.0047 \\
\hline $\mathrm{BMI}\left(\mathrm{kg} / \mathrm{m}^{2}\right)$ & $34.1[30.4 ; 39.5]$ & $33.2[30.3 ; 38.3]$ & 0.8851 & $32.6[31.8 ; 36.5]$ & $32[30.6 ; 35.3]$ & $<0.0001$ & 0.0045 \\
\hline Waist circumference (cm) & $113[103 ; 124]$ & $112[102 ; 121]$ & 0.2623 & $114[103 ; 120]$ & $113[101 ; 117]$ & 0.0360 & 0.4266 \\
\hline \multicolumn{8}{|l|}{ Biological parameters } \\
\hline Fasting blood glucose $(\mathrm{mmol} / \mathrm{L})$ & $9.22 \pm 2.49$ & $8.74 \pm 2.42$ & 0.2516 & $9.59 \pm 2.44$ & $7.35 \pm 1.73$ & $<0.0001$ & 0.0063 \\
\hline $\operatorname{HbA1c}(\%)$ & $8.1 \pm 1.1$ & $7.8 \pm 0.9$ & 0.0605 & $8.1 \pm 1.0$ & $7.3 \pm 0.6$ & $<0.0001$ & 0.0033 \\
\hline Total cholesterol (g/L) & $1.72 \pm 0.40$ & $1.68 \pm 0.35$ & 0.5340 & $1.57 \pm 0.37$ & $1.57 \pm 0.44$ & 0.8655 & 0.5111 \\
\hline HDL-cholesterol (g/L) & $0.41[0.32 ; 0.45]$ & $0.42[0.33 ; 0.5]$ & 0.7394 & $0.40[0.36 ; 0.50]$ & $0.46[0.38 ; 0.53]$ & 0.0123 & 0.1359 \\
\hline LDL-cholesterol (g/L) & $0.92 \pm 0.25$ & $0.92 \pm 0.29$ & 0.9668 & $0.83 \pm 0.30$ & $0.84 \pm 0.34$ & 0.6199 & 0.7940 \\
\hline Triglycerides (g/L) & $1.56[1.11 ; 2.13]$ & $1.47[1.01 ; 2.35]$ & 0.9881 & $1.48[0.94 ; 1.97]$ & $1.27[0.84 ; 1.54]$ & 0.0451 & 0.6447 \\
\hline Uricemia $(\mu \mathrm{mol} / \mathrm{L})$ & $284.6 \pm 81.1$ & $296.0 \pm 87.8$ & 0.3391 & $301.8 \pm 82.3$ & $265.7 \pm 67.1$ & 0.0052 & 0.0062 \\
\hline Ferritinemia & $65.0[15.0 ; 158.9]$ & $60.2[16.4 ; 126.5]$ & 0.8462 & $75.7[34.3 ; 133.0]$ & $48.6[19.9 ; 92.3]$ & $<0.0001$ & 0.0445 \\
\hline hs-CRP (mg/L) & $3.7[1.8 ; 5.1]$ & $3.4[1.7 ; 6.1]$ & 0.1058 & $2.3[1.0 ; 5.6]$ & $2.3[1 ; 5.3]$ & 0.1349 & 0.2044 \\
\hline PAI-1 (UI/L) & $41[11 ; 79]$ & $36.5[10.2 ; 72]$ & 0.3938 & 24 [15.2;39.2] & $16[8.5 ; 28.5]$ & 0.1396 & 0.4318 \\
\hline AST (UI/L) & $23[20 ; 32]$ & $24[18 ; 33]$ & 0.6062 & $23[20 ; 33]$ & $23[19 ; 32]$ & 0.2339 & 0.1219 \\
\hline ALT (UI/L) & $33[23 ; 54]$ & $34[23 ; 44]$ & 0.3178 & $30[25 ; 42]$ & $28[18 ; 44]$ & 0.0302 & 0.0705 \\
\hline GGT (UI/L) & $42[30 ; 76]$ & $43[26 ; 63]$ & 0.3698 & $36[26 ; 47]$ & $28[24 ; 42]$ & 0.0002 & 0.2219 \\
\hline Hematocrit & $0.38[0.35 ; 0.41]$ & $0.38[0.36 ; 0.43]$ & 0.5520 & $0.40[0.37 ; 0.43]$ & $0.43[0.40 ; 0.46]$ & $<0.0001$ & 0.0001 \\
\hline Reticulocytes (\%) & $1.4[1.2 ; 1.8]$ & $1.5[1.1 ; 1.7]$ & 0.4463 & $1.5[1.2 ; 1.8]$ & $1.3[1.2 ; 1.6]$ & 0.0030 & 0.0027 \\
\hline $\mathrm{EPO}(\mathrm{mU} / \mathrm{mL})$ & $13[8.5 ; 18]$ & $11[8.2 ; 16.5]$ & 0.1709 & $8.7[4.9 ; 14]$ & $9.2[5.9 ; 13.7]$ & 0.1789 & 0.0412 \\
\hline Glycosuria (mmol/L) & $0.75[0.31 ; 3.13]$ & $1.06[0.36 ; 5.72]$ & 0.6434 & $1.13[0.56 ; 12.83]$ & $193.3[125.4 ; 256.7]$ & $<0.0001$ & $<0.0001$ \\
\hline \multicolumn{8}{|l|}{ MRI parameters } \\
\hline Ejection fraction (\%) & $63.7 \pm 8.3$ & $63.3 \pm 7.7$ & 0.6598 & $63.1 \pm 8.2$ & $63.3 \pm 7.2$ & 0.8515 & 0.6515 \\
\hline LVM (g) & $129[107 ; 149]$ & $124[107 ; 151]$ & 0.2217 & $117[93 ; 150]$ & $110[97 ; 142]$ & 0.5153 & 0.9707 \\
\hline LVMi (g) & $64[59 ; 72]$ & $64[57 ; 69]$ & 0.1429 & $58[51 ; 66]$ & $58[52 ; 64]$ & 0.3802 & 0.9545 \\
\hline E/A ratio & $1.26[1.07 ; 1.49]$ & $1.17[0.85 ; 1.44]$ & 0.3289 & $1.14[0.90 ; 1.71]$ & $1.08[0.92 ; 1.62]$ & 0.5619 & 0.3923 \\
\hline
\end{tabular}

BMI body mass index; PAI-1 plasminogen activator inhibitor-1; EPO: erythropoietin; LVEF left ventricular ejection fraction; LVM left ventricular mass; LVMi left ventricular mass indexed to body surface area

Twelve weeks of empagliflozin treatment did not modulate epicardial fat volume in humans

Recently, there has been growing interest in how SGLT2 inhibitors reduce cardiovascular outcomes in patients with T2D [19]. However, few mechanisms of action have been robustly investigated. Novel multiple cardiac imaging modalities combining ${ }^{1} \mathrm{H}$-MRS and ${ }^{1} \mathrm{P}-\mathrm{MRS}$ enabled us to test the appealing hypothesis that empagliflozin could modulate ectopic fat stores such as cardiac ectopic fat (epicardial, myocardial fat), pancreatic fat or myocardial energetics (as assessed by PCr/ATP ratio). Epicardial fat volume mobilization was the primary endpoint of the human study.

Epicardial adipose tissue (EAT) is a visceral fat deposit located between the visceral pericardium and the myocardium. However, unlike visceral abdominal adipose tissue, it has predominantly local effects, making up less than $1 \%$ of total fat mass [8]. EAT has a unique anatomical proximity to the heart. As there is no dividing fascia plane between EAT, coronary arteries adventitia and myocardium, there can be cellular crosstalk and functional relationships between ectopic adipocytes, myocytes and cells of the vascular wall. Free fatty acids (FFAs), proinflammatory cytokines (IL-6, IL-1 $\beta$, IL-8, sPLA2-IIA), vasoactive entities (angiotensin) adipofibrokines (Activin-A) and growth factors (TNF- $\alpha, \mathrm{VEGF)}$ are likely to diffuse from EAT to the lumen of coronary arteries. The amount of EAT has been shown to predict fatal and non-fatal cardiac outcomes at 8 years in highrisk cardiovascular patients and has been associated 


\section{(See figure on next page.)}

Fig. 3 Changes in myocardial energetics, metabolic profile and ectopic fat stores at 12 weeks of treatment in humans. a Changes in glycosuria, blood ketone body levels, hematocrit and PCr/ATP ratio obtained by ${ }^{31} \mathrm{P}$-magnetic resonance spectroscopy in placebo and EMPA group. b Liver and pancreatic fat content assessed with ${ }^{1} \mathrm{H}$-magnetic resonance spectroscopy change between baseline and 12 weeks in placebo and EMPA group. $\mathbf{c}$ Visceral and subcutaneous abdominal fat change measured at L4-L5 intervertebral disk level; Epicardial fat volume assessed on cine MRI short axis slices and myocardial fat assessed with ${ }^{1} \mathrm{H}$-magnetic resonance spectroscopy at the interventricular septum with a single-voxel PRESS sequence. Placebo $(n=16)$, EMPA $(n=18)$. Data represent mean values \pm SEM. Differences between baseline and 12 weeks were determined by paired $t$-test/ Wilcoxon test and differences between groups with ANOVA; ${ }^{*} p<0.05,{ }^{* *} p<0.01,{ }^{* * *} p<0.001$

with plaque vulnerability, myocardial infarction, unstable angina, coronary flow reserve, and stent restenosis $[31,32]$. This study showed no effect of empagliflozin on epicardial fat volume in high-risk patients with T2D and normal LVEF. Epicardial fat was not measured in the mouse study because rodents have no EAT [8]. Few studies have evaluated the effect of SGLT2 inhibitors on epicardial fat volume [33-36]. These studies were observational, open-label, single-arm with multiple imaging modalities, and yielded conflicting results. To our knowledge, this is the first randomized, placebo-controlled clinical trial to examine the effect of empagliflozin on epicardial fat volume assessed with the more accurate non-invasive method (3 T MRI) in T2D subjects. In a lipodystrophic mouse model of diabetic cardiomyopathy (seipin-knockout mice with no lipotoxic hallmarks and no intramyocardial lipid accumulation), Joubert et al. showed a beneficial effect of dapagliflozin on cardiac dysfunction with improvement of myocardial glucose uptake, and decreased activation of the hexosamine pathway (O-GlcNacylated protein levels), suggesting that SGLT2 inhibitors could act on glucotoxicity rather than lipotoxicity mechanisms. Interestingly, whereas the heart does not express SGLT2, Diaz-Rodriguez et al. reported SGLT2 expression in epicardial adipose tissue, increased in vitro glucose uptake and GLUT4, reduced secretion of proinflammatory chemokines, and improved differentiation of EAT cells with dapagliflozin in EAT of patients undergoing open heart surgery $(30 \% \mathrm{~T} 2 \mathrm{D}$, and $50 \%$ with CAD) [37]. The lack of change in epicardial fat volume observed in our study does not imply no change in molecular characteristics of EAT with SGLT2 inhibition that could influence heart function. This change in EAT phenotype might contribute at least to a decreased adverse remodeling of the overloaded diabetic heart.

\section{Twelve weeks of empagliflozin treatment did not change myocardial fat or left ventricular function}

We observed no change in LV mass or E/A ratio at 12 weeks between groups, in contrast to the EMPAHEART study, which reported a significant decrease in LV mass indexed to body surface area in patients with T2D and CAD and treated with empagliflozin after
6 months, suggesting that the improvement of LV function could be delayed and may not be sufficient to explain early cardiovascular benefits [38]. In $\mathrm{db} / \mathrm{db}$ mice, Moellmann et al. showed that improvement of diastolic function could be mediated by reduced spontaneous diastolic sarcoplasmic reticulum calcium release, independently of changes in cardiac ketone metabolism [39]. Our results are consistent with the open-label trial of Hsu et al, who found no effect of 6 months of empagliflozin treatment on LV function, pericardial myocardial fat, myocardial fibrosis or LV mass [34]. Likewise, this study reported no effect of empagliflozin on myocardial triglyceride content at 12 weeks.

\section{Twelve weeks of empagliflozin treatment did not change pancreatic fat content}

SGLT-2 inhibitors are known to improve $\beta$ cell function and insulin sensitivity in T2D [40]. Previous studies have suggested that increased pancreatic fat deposition (nonalcoholic fatty pancreas disease) could contribute to $\beta$ cell dysfunction and could sustain insulin resistance [41]. We previously demonstrated that patients with T2D have an increased ectopic fat storage in the pancreas compared to obese nondiabetics and lean subjects [42]. To our knowledge, this study is innovative in that it is the first one reporting no effect of an SGLT2 inhibitor on pancreatic fat content in humans, as measured with ${ }^{1} \mathrm{H}$-MRS. With this technique, we could not differentiate intralobular or parenchymal fat in humans in vivo, as previously described by others with modified DIXON (mDIXON) water images and mDIXON MRI fat fraction method [43]. We did not assess pancreatic fat in mice because of challenging pancreas localization with ${ }^{1} \mathrm{H}$-MRS. This exploratory analysis now needs to be confirmed in larger long-term clinical trials dedicated to pancreas morphological features and endocrine pancreas function $[44,45]$.

\section{Twelve weeks of empagliflozin treatment greatly reduced liver fat content in mice and humans}

This study showed a significant effect of empagliflozin on liver fat in mice and humans. In Otsuka Long-Evans Tokushima fatty rats, Kim et al. [46] had already shown significant reduction of hepatic steatosis with empagliflozin and a decrease in lipogenesis and gluconeogenesis 


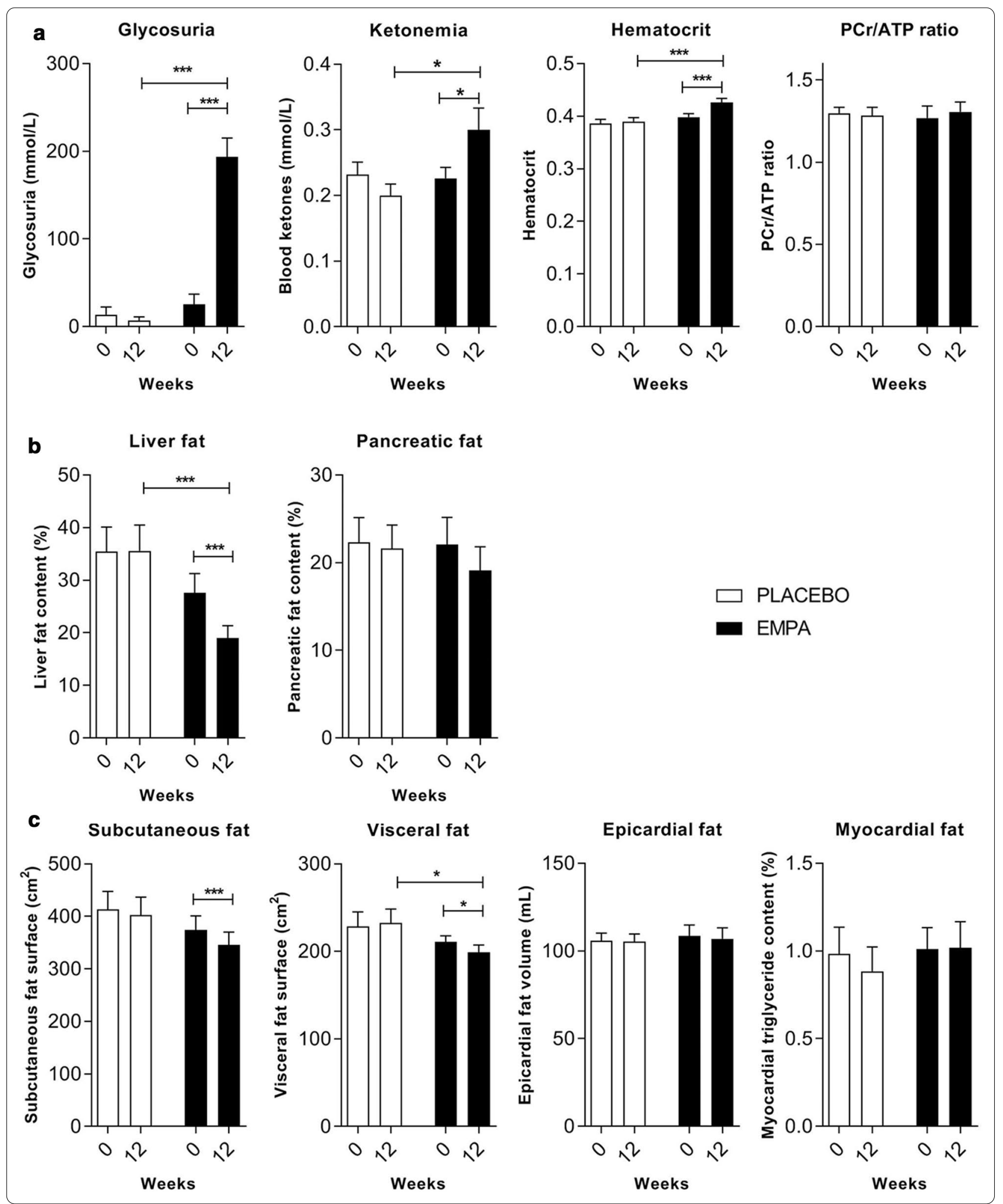


gene expression, and increased SIRT1 and AMPK expression in the liver. In an Amylin liver non-alcoholic steato-hepatitis (NASH) model, Honda et al. [47] also demonstrated that ipragliflozin $(40 \mathrm{mg} / \mathrm{kg} / \mathrm{d})$ for 8 weeks improved insulin resistance and liver injury, decreased hepatic lipid content, areas of fibrosis and also increased lipid outflow from the liver at 8 weeks of treatment. Other animal studies point to a reduction in hepatic inflammatory cytokines and oxidative stress or an increase in ketone body metabolism with SGLT-2 inhibitors [48]. However, this mouse study showed no effect of empagliflozin on genes coding for enzymes involved in KB metabolism. An effect of empagliflozin on liver fat content may be also explained by a negative energy balance via substantial urine glucose loss and with an increase in fatty acid oxidation that might be promoted by a decreased insulin/glucagon ratio [49]. This human study revealed that $96 \%$ of the studied population had liver steatosis (as defined by LFC $>5 \%$ ) at baseline, underlining the extreme prevalence of NAFLD in T2D even in patients with normal transaminases levels $(12,16,26)$. The effect of empagliflozin on liver fat content occurred in parallel to the decline in body weight during SGLT2 inhibitor treatment. We and others previously demonstrated that liver fat is the most rapidly mobilized ectopic fat store during nutritional, medical or surgery-induced weight loss interventions (27). Also, the weight reduction $\geq 5 \%$ observed in only $38 \%$ of patients on EMPA suggests that even minor weight loss might have an impact on liver fat content. These results are consistent with the E-LIFT open-label trial in which empagliflozin $10 \mathrm{mg}$ significantly decreased MRI-derived liver proton density fat fraction (PDFF) compared to patients not treated with EMPA (28), and to the results of Kahl et al. [50] who reported a significant effect of empagliflozin $25 \mathrm{mg}$ compared to placebo in T2D patients with excellent glycemic control and short disease duration. Other studies have yielded similar results with dapagliflozin [51, 52], ipragliflozin [53], and luseogliflozin [54], and a recent Japanese study showed histopathological improvement with canagliflozin (25), suggesting that this beneficial effect could be a class effect. However, few studies used ${ }^{1} \mathrm{H}$-MRS, one of the most accurate and reproducible non-invasive techniques for NAFLD assessment in patients with obesity and T2D [55]. We observed a significant parallel decrease in visceral fat accumulation $(-6 \%)$ in the EMPA group compared to placebo, which is in agreement with a recent meta-analysis [48]. Taken together, these results suggest that SGLT-2 inhibitors could have a therapeutic effect on NAFLD and visceral adiposity in patients with T2DM.
Twelve weeks of empagliflozin treatment did not change high-energy phosphate metabolism in humans

In this study, we also tested the myocardial energetics hypothesis in humans in vivo and investigated whether an increase in plasma ketones by empagliflozin was associated with an increase in rest cardiac PCr/ATP using ${ }^{31} \mathrm{P}$ MRS. Remarkably, we found no effect of empagliflozin on PCr/ATP ratio, despite a significant increase in blood KB levels, while PCr/ATP was significantly decreased in T2D patients compared to healthy controls (data not shown). To our knowledge, although we did not assess myocardial substrates disposal, this is the first study to report the effect of an SGLT2 inhibitor on PCr/ATP in humans. In isolated hearts of healthy mice, Uthman et al. [56] showed no effect of empagliflozin, dapagliflozin, or canagliflozin on PCr/ATP, but found inhibition of cardiac $\mathrm{NA}+/ \mathrm{H}+$ exchanger and significant reduction in cardiac cytosolic $\mathrm{Na}+$ concentration, suggesting another mechanism by which SGLT2 inhibitors could exert beneficial effects in heart failure. By contrast, Santos-Gallego et al. [49] reported increased myocardial ATP content and enhanced myocardial work efficiency in empagliflozintreated nondiabetic pigs. Abdurrachim et al. [57] showed a $45 \%$ increase in cardiac PCr/ATP in EMPA treated $d b /$ $d b$ mice compared to placebo-treated $d b / d b$ mice. Verma et al. using isolated working heart from $d b / d b$ mice, demonstrated that empagliflozin increased cardiac ATP production by $30 \%$ and prevented heart failure. This effect was due to an increase in glucose and fatty acid oxidation, but with no change in ketone oxidation, suggesting that empagliflozin enhances the cardiac energy pool by increasing fuel supply to the failing heart, more than supplying the heart with a more efficient source of fuel $[4,58]$. In our study, significant increase in hematocrit and EPO was observed in the EMPA group compared to placebo, possibly contributing to enhanced myocardial tissue oxygen delivery. Altogether, our results do not support an early change in myocardial energetics at rest in overweight T2D patients with normal LVEF treated with empagliflozin for 12 weeks. Whether empagliflozin can improve cardiac energetics in the context of increased workload such as was observed in obese subjects with weight loss needs to be evaluated [59].

The strengths of this study lie in the use of preclinical and clinical models and a randomized controlled trial design with concomitant measurement of several ectopic fat stores using novel multiple cardiac imaging modalities.

\section{Limits}

We also acknowledge some limitations, such as that myocardial energetics was only evaluated in humans, in a subgroup of patients, only at rest, and in an exploratory 
analysis. An effect of empaglifozin on myocardial energetics during stress or exercise or later on can therefore not be excluded. We acknowledge that the intervention period may have been too short to observe a significant change in epicardial, myocardial fat and myocardial energetics. Longer interventional studies are now needed to draw definite conclusions on the effect of empagliflozin on cardiac ectopic fat stores. However, the sensitivity of ${ }^{31} \mathrm{P}$-MRS to detect changes in tissue energetics was high, as we were able to measure a significant decrease in $\mathrm{PCr} /$ ATP in T2D versus healthy controls. The sample size was perhaps also insufficiently powered to see an effect, but these results can serve to design future prospective studies. We could not assess PCr/ATP in mice for want of a ${ }^{31} \mathrm{P}$ surface heart coil adapted to small animals. We also did not assess pancreatic fat in mice because of the wellknown difficulty of delineating the pancreas in rodents with MRI. Finally, we did not assess myocardial substrates disposal in this study, but a recent study showed a $>50 \%$ decrease in myocardial glucose uptake after 4 weeks of treatment with empagliflozin in T2D individuals [18].

In conclusion, this randomized controlled trial showed that empagliflozin promptly reduced liver and visceral fat compared to placebo without changing cardiac ectopic fat or myocardial energetics. This study combined multiple novel cardiac imaging modalities such as ${ }^{1} \mathrm{H}$-MRS and ${ }^{31} \mathrm{P}$-MRS for ectopic fat and cardiac metabolism quantification, which are innovative, but validated and robust non-invasive MRI techniques.

\section{Conclusions}

The study suggests that SGLT-2 inhibitors are useful agents for improving NAFLD, which often coexists with type 2 diabetes. To investigate whether SGLT-2 inhibitors also lead to improvement in steatohepatitis NASH and/or liver fibrosis would require randomized histopathological studies. In this study, no effect of empagliflozin on epicardial, myocardial, pancreatic fat or $\mathrm{PCr} /$ ATP ratio was evidenced at 12 weeks. The absence of any effect of empaglifozin on pancreatic and cardiac steatosis strengthens the tissue-specific mobilization of ectopic fat stores with therapeutic interventions. Further experimental and clinical studies are now needed to elucidate how SGLT2 inhibitors exert these impressive cardiovascular effects.

\section{Supplementary Information}

The online version contains supplementary material available at https://doi. org/10.1186/s12933-021-01237-2.

Additional file 1. Supplementary material and methods for animals.

\section{Acknowledgments}

We thank Eric Gianfranceschi for his technical support in Oil-red-O mice experiments, all the staff of CEMEREM and CRMBM for their help in cardiovascular imaging modalities and patients for their participation in this study.

\section{Authors' contributions}

Not applicable.

\section{Funding}

This work was supported by a grant from Boehringer-Ingelheim.

\section{Availability of data and materials}

The datasets used and/or analyzed during the current study are available from the corresponding author on reasonable request.

\section{Ethics approval and consent to participate}

All animal procedures were approved by the ethics committee of Aix-Marseille University and complied with the European Convention for the protection of animals used for experimental purposes. The local ethics committee (CPP lle-de-France VII/ANSM) approved all trial procedures in compliance with the Declaration of Helsinki. The EMPACEF clinical trial was registered at clinicaltrials.gov (NCT03118336).

\section{Consent for publication}

Not applicable.

\section{Competing interests}

The authors declare that they have no competing interests.

\section{Author details}

${ }^{1}$ Aix Marseille Univ, INSERM, INRAE, C2VN Marseille, France. ${ }^{2}$ Department of Endocrinology, Metabolic Diseases and Nutrition, Pôle ENDO, APHM, Hôpital Nord, Chemin Des Bourrely, 13915 Marseille cedex 20, France. ${ }^{3}$ Aix Marseille Univ, CNRS, CRMBM, Marseille, France. ${ }^{4}$ Assistance-Publique Hôpitaux de Marseille, Medical Evaluation Department, CIC-CPCET, 13005 Marseille, France. ${ }^{5}$ Support Unit for Clinical Research and Economic Evaluation, Assistance Publique-Hôpitaux de Marseille, 13385 Marseille, France. ${ }^{6}$ Aix-Marseille Univ, EA 3279 CEReSS-Health Service Research and Quality of Life Center, Marseille, France.

Received: 11 December 2020 Accepted: 1 February 2021

Published online: 01 March 2021

\section{References}

1. Zinman B, Wanner C, Lachin JM, Fitchett D, Bluhmki E, Hantel S, et al. Empagliflozin, cardiovascular outcomes, and mortality in type 2 diabetes. N Engl J Med. 2015;373:2117-28.

2. Neal B, Perkovic V, Mahaffey KW, de Zeeuw D, Fulcher G, Erondu N, et al. Canagliflozin and cardiovascular and renal events in type 2 diabetes. $\mathrm{N}$ Engl J Med. 2017;377:644-57.

3. Wiviott SD, Raz I, Bonaca MP, Mosenzon O, Kato ET, Cahn A, et al. Dapagliflozin and cardiovascular outcomes in type 2 diabetes. N Engl J Med. 2019;380:347-57.

4. Lopaschuk GD, Verma S. Mechanisms of cardiovascular benefits of sodium glucose co-transporter 2 (SGLT2) Inhibitors: A State-Of-The-Art Review. JACC Basic Transl Sci. 2020;5:632-44.

5. Lee H-C, Shiou Y-L, Jhuo S-J, Chang C-Y, Liu P-L, Jhuang W-J, et al. The sodium-glucose co-transporter 2 inhibitor empagliflozin attenuates cardiac fibrosis and improves ventricular hemodynamics in hypertensive heart failure rats. Cardiovasc Diabetol. 2019;18:45.

6. Koyani CN, Plastira I, Sourij H, Hallström S, Schmidt A, Rainer PP, et al. Empagliflozin protects heart from inflammation and energy depletion via AMPK activation. Pharmacol Res. 2020;158:104870.

7. Park S-H, Farooq MA, Gaertner S, Bruckert C, Qureshi AW, Lee H-H, et al. Empagliflozin improved systolic blood pressure, endothelial dysfunction and heart remodeling in the metabolic syndrome ZSF1 rat. CardiovasC Diabetol. 2020;19:19. 
8. Gaborit B, Sengenes C, Ancel P, Jacquier A, Dutour A. Role of epicardial adipose tissue in health and disease: a matter of fat? Compr Physiol. 2017;7:1051-82

9. Perseghin G, Lattuada G, De Cobelli F, Esposito A, Belloni E, Ntali G, et al. Increased mediastinal fat and impaired left ventricular energy metabolism in young men with newly found fatty liver. Hepatol Baltim Md. 2008:47:51-8

10. Gaborit B, Kober F, Jacquier A, Moro PJ, Cuisset T, Boullu S, et al. Assessment of epicardial fat volume and myocardial triglyceride content in severely obese subjects: relationship to metabolic profile, cardiac function and visceral fat. Int J Obes. 2012;36:422-30.

11. Gaborit B, Jacquier A, Kober F, Abdesselam I, Cuisset T, Boullu-Ciocca S, et al. Effects of bariatric surgery on cardiac ectopic fat: lesser decrease in epicardial fat compared to visceral fat loss and no change in myocardial triglyceride content. J Am Coll Cardiol. 2012;60:1381-9.

12. Gupta A, Akki A, Wang Y, Leppo MK, Chacko VP, Foster DB, et al. Creatine kinase-mediated improvement of function in failing mouse hearts provides causal evidence the failing heart is energy starved. J Clin Invest. 2012;122:291-302.

13. Bottomley PA, Panjrath GS, Lai S, Hirsch GA, Wu K, Najjar SS, et al. Metabolic rates of ATP transfer through creatine kinase (CK Flux) predict clinical heart failure events and death. Sci Transl Med. 2013;5:215re3.

14. Neubauer S. The failing heart-an engine out of fuel. N Engl J Med. 2007:356:1140-51.

15. Neubauer S, Horn M, Cramer M, Harre K, Newell JB, Peters W, et al. Myocardial phosphocreatine-to-ATP ratio is a predictor of mortality in patients with dilated cardiomyopathy. Circulation. 1997;96:2190-6.

16. Ferrannini E, Mark M, Mayoux E. CV Protection in the EMPA-REG OUTCOME trial: a "thrifty substrate" hypothesis. Diabetes Care. 2016:39:1108-14.

17. Mudaliar S, Alloju S, Henry RR. Can a shift in fuel energetics explain the beneficial cardiorenal outcomes in the EMPA-REG OUTCOME Study? A Unify Hypothesis Diabetes Care. 2016;39:1115-22.

18. Lauritsen KM, Nielsen BRR, Tolbod LP, Johannsen M, Hansen J, Hansen TK, et al. SGLT2 inhibition does not affect myocardial fatty acid oxidation or uptake, but reduces myocardial glucose uptake and blood flow in individuals with type 2 diabetes- a randomized double-blind PlaceboControlled Crossover Trial. Diabetes. 2020. https://doi.org/10.2337/ db20-0921.

19. Lee MMY, Petrie MC, MCMurray JJV, Sattar N. How Do SGLT2 (SodiumGlucose Cotransporter 2) Inhibitors and GLP-1 (Glucagon-Like Peptide-1) receptor agonists reduce cardiovascular outcomes?: completed and ongoing mechanistic trials. Arterioscler Thromb Vasc Biol. 2020;40:506-22.

20. Abdesselam I, Pepino P, Troalen T, Macia M, Ancel P, Masi B, et al. Time course of cardiometabolic alterations in a high fat high sucrose diet mice model and improvement after GLP-1 analog treatment using multimodal cardiovascular magnetic resonance. J Cardiovasc Magn Reson Off J Soc Cardiovasc Magn Reson. 2015;17:95

21. World Health Organization. Classification of Diabetes Mellitus. 2019. https ://apps.who.int/iris/rest/bitstreams/1233344/retrieve

22. American Diabetes Association. 5. Facilitating behavior change and well-being to improve health outcomes: standards of medical care in diabetes-2020. Diabetes Care 2020:543:48-65.

23. Maurice F, Gaborit B, Vincentelli C, Abdesselam I, Bernard M, Graillon $T$, et al. Cushing syndrome is associated with subclinal left ventricular dysfunction and increased epicardial adipose tissue. J Am Coll Cardiol. 2018:22:1551-67 (epub ahead of print)

24. Dutour A, Abdesselam I, Ancel P, Kober F, Mrad G, Darmon P, et al. Exenatide decreases liver fat content and epicardial adipose tissue in patients with obesity and type 2 diabetes: a prospective randomized clinical trial using magnetic resonance imaging and spectroscopy. Diabetes Obes Metab. 2016;18:882-91.

25. Bottomley PA. MR spectroscopy of the human heart: the status and the challenges. Radiology. 1994;191:593-612.

26. Le Fur Y, Nicoli F, Guye M, Confort-Gouny S, Cozzone PJ, Kober F. Gridfree interactive and automated data processing for MR chemical shift imaging data. Magma N Y N. 2010;23:23-30.

27. Vanhamme L, van den Boogaart A, Van Huffel S. Improved method for accurate and efficient quantification of MRS data with use of prior knowledge. J Magn Reson San Diego Calif. 1997;129:35-43.
28. El-Sharkawy A-M, Schär M, Ouwerkerk R, Weiss RG, Bottomley PA. Quantitative cardiac 31P spectroscopy at 3 Tesla using adiabatic pulses. Magn Reson Med. 2009:61:785-95.

29. Schär M, El-Sharkawy A-MM, Weiss RG, Bottomley PA. Triple repetition time saturation transfer (TRiST) 31P spectroscopy for measuring human creatine kinase reaction kinetics. Magn Reson Med. 2010;63:1493-501.

30. Clarke WT, Peterzan MA, Rayner JJ, Sayeed RA, Petrou M, Krasopoulos G, et al. Localized rest and stress human cardiac creatine kinase reaction kinetics at 3 T. NMR Biomed. 2019;32:e4085.

31. lacobellis G. Local and systemic effects of the multifaceted epicardial adipose tissue depot. Nat Rev Endocrinol. 2015;11:363-71.

32. Mahabadi AA, Berg MH, Lehmann N, Kälsch H, Bauer M, Kara K, et al. Association of epicardial fat with cardiovascular risk factors and incident myocardial infarction in the general population: the Heinz Nixdorf Recall Study. J Am Coll Cardiol. 2013;61:1388-95.

33. Sato T, Aizawa Y, Yuasa S, Kishi S, Fuse K, Fujita S, et al. The effect of dapagliflozin treatment on epicardial adipose tissue volume. Cardiovasc Diabetol. 2018;17:6.

34. Hsu J-C, Wang C-Y, Su M-YM, Lin L-Y, Yang W-S. Effect of empagliflozin on cardiac function, adiposity, and diffuse fibrosis in patients with type 2 diabetes mellitus. Sci Rep. 2019;9:15348.

35. Braha A, Timar B, Diaconu L, Lupusoru R, Vasiluta L, Sima A, et al. Dynamics of epicardiac fat and heart function in type 2 diabetic patients initiated with sglt-2 inhibitors. Diabetes Metab Syndr Obes Targets Ther. 2019;12:2559-66.

36. Fukuda T, Bouchi R, Terashima M, Sasahara Y, Asakawa M, Takeuchi T, et al. Ipragliflozin reduces epicardial fat accumulation in non-obese type 2 diabetic patients with visceral obesity: a pilot study. Diabetes Ther Res Treat Educ Diabetes Relat Disord. 2017;8:851-61.

37. Díaz-Rodríquez E, Agra RM, Fernández ÁL, Adrio B, García-Caballero T, González-Juanatey JR, et al. Effects of dapagliflozin on human epicardial adipose tissue: modulation of insulin resistance, inflammatory chemokine production, and differentiation ability. Cardiovasc Res. 2018;114:336-46.

38. Verma S, Mazer CD, Yan AT, Mason T, Garg V, Teoh H, et al. Effect of Empagliflozin on left ventricular mass in patients with type 2 diabetes mellitus and coronary artery disease: the EMPA-HEART CardioLink-6 randomized clinical trial. Circulation. 2019;140:1693-702.

39. Moellmann J, Klinkhammer BM, Droste P, Kappel B, Haj-Yehia E, Maxeiner $\mathrm{S}$, et al. Empagliflozin improves left ventricular diastolic function of $\mathrm{db} / \mathrm{db}$ mice. Biochim Biophys Acta Mol Basis Dis. 2020;1866:165807.

40. Ferrannini E, Muscelli E, Frascerra S, Baldi S, Mari A, Heise T, et al. Metabolic response to sodium-glucose cotransporter 2 inhibition in type 2 diabetic patients. J Clin Invest. 2014;124:499-508.

41. Sattar N, Gill JMR. Type 2 diabetes as a disease of ectopic fat? BMC Med. 2014;12:123.

42. Gaborit B, Abdesselam I, Kober F, Jacquier A, Ronsin O, Emungania O, et al. Ectopic fat storage in the pancreas using $1 \mathrm{H}$-MRS: importance of diabetic status and modulation with bariatric surgery-induced weight loss. Int J Obes. 2005;2015(39):480-7.

43. Begovatz P, Koliaki C, Weber K, Strassburger K, Nowotny B, Nowotny P, et al. Pancreatic adipose tissue infiltration, parenchymal steatosis and beta cell function in humans. Diabetologia. 2015;58:1646-55.

44. Al-Mrabeh A, Hollingsworth KG, Shaw JAM, McConnachie A, Sattar $\mathrm{N}$, Lean MEJ, et al. 2-year remission of type 2 diabetes and pancreas morphology: a post-hoc analysis of the DiRECT open-label, clusterrandomised trial. Lancet Diabetes Endocrinol. 2020;8:939-48.

45. Al-Mrabeh A, Hollingsworth KG, Steven S, Taylor R. Morphology of the pancreas in type 2 diabetes: effect of weight loss with or without normalisation of insulin secretory capacity. Diabetologia. 2016;59:1753-9.

46. Kim J-W, Lee Y-J, You Y-H, Moon MK, Yoon K-H, Ahn Y-B, et al. Effect of sodium-glucose cotransporter 2 inhibitor, empagliflozin, and a-glucosidase inhibitor, voglibose, on hepatic steatosis in an animal model of type 2 diabetes. J Cell Biochem. 2018;120:8534-46.

47. Honda Y, Imajo K, Kato T, Kessoku T, Ogawa Y, Tomeno W, et al. The Selective SGLT2 Inhibitor Ipragliflozin Has a Therapeutic Effect on Nonalcoholic Steatohepatitis in Mice. PLoS ONE. 2016;11:e0146337.

48. Xing B, Zhao Y, Dong B, Zhou Y, Lv W, Zhao W. Effects of sodium-glucose cotransporter 2 inhibitors on non-alcoholic fatty liver disease in patients 
with type 2 diabetes: a meta-analysis of randomized controlled trials. J Diabetes Investig. 2020;11:1238-47.

49. Santos-Gallego CG, Requena-Ibanez JA, San Antonio R, Ishikawa K, Watanabe S, Picatoste B, et al. Empagliflozin ameliorates adverse left ventricular remodeling in Nondiabetic heart failure by enhancing myocardial energetics. J Am Coll Cardiol. 2019;73:1931-44.

50. Kahl S, Gancheva S, Straßburger K, Herder C, Machann J, Katsuyama H, et al. Empagliflozin effectively lowers liver fat content in well-controlled type 2 diabetes: a randomized, double-blind, phase 4. Placebo-Controlled Trial Diabetes Care. 2020;43:298-305.

51. Eriksson JW, Lundkvist $P$, Jansson P-A, Johansson L, Kvarnström M, Moris $L$, et al. Effects of dapagliflozin and n-3 carboxylic acids on non-alcoholic fatty liver disease in people with type 2 diabetes: a double-blind randomised placebo-controlled study. Diabetologia. 2018;61:1923-34.

52. Latva-Rasku A, Honka M-J, Kullberg J, Mononen N, Lehtimäki T, Saltevo J, et al. The SGLT2 Inhibitor Dapagliflozin reduces liver fat but does not affect tissue insulin sensitivity: a randomized, double-blind, placebo-controlled study with 8-week treatment in type 2 diabetes patients. Diabetes Care. 2019;42:931-7.

53. Ito D, Shimizu S, Inoue K, Saito D, Yanagisawa M, Inukai K, et al. Comparison of Ipragliflozin and pioglitazone effects on nonalcoholic fatty liver disease in patients with type 2 diabetes: a randomized, 24-week, openlabel Active-Controlled Trial. Diabetes Care. 2017;40:1364-72.

54. Shibuya T, Fushimi N, Kawai M, Yoshida Y, Hachiya H, Ito S, et al. Luseogliflozin improves liver fat deposition compared to metformin in type 2 diabetes patients with non-alcoholic fatty liver disease: a prospective randomized controlled pilot study. Diabetes Obes Metab. 2018;20:438-42.

55. European Association for the Study of the Liver (EASL), European Association for the Study of Diabetes (EASD), European Association for the Study of Obesity (EASO). EASL-EASD-EASO Clinical Practice Guidelines for the management of non-alcoholic fatty liver disease. J Hepatol. 2016;64:1388-402.

56. Uthman L, Baartscheer A, Bleijlevens B, Schumacher CA, Fiolet JWT, Koeman A, et al. Class effects of SGLT2 inhibitors in mouse cardiomyocytes and hearts: inhibition of $\mathrm{Na}+/ \mathrm{H}+$ exchanger, lowering of cytosolic $\mathrm{Na}+$ and vasodilation. Diabetologia. 2018;61:722-6.

57. Abdurrachim D, Manders E, Nicolay K, Mayoux E, Prompers JJ. Single dose of empagliflozin increases in vivo cardiac energy status in diabetic $\mathrm{db} / \mathrm{db}$ mice. Cardiovasc Res. 2018;114:1843-4.

58. Verma S, Rawat S, Ho KL, Wagg CS, Zhang L, Teoh H, et al. Empagliflozin increases cardiac energy production in diabetes: novel translational insights into the heart failure benefits of SGLT2 inhibitors. JACC Basic Transl Sci. 2018;3:575-87.

59. Rayner JJ, Peterzan MA, Watson WD, Clarke WT, Neubauer S, Rodgers CT, et al. Myocardial energetics in obesity: enhanced ATP delivery through creatine kinase with blunted stress response. Circulation. 2020;141:1152-63.

60. Piepoli MF, Hoes AW, Agewall S, Albus C, Brotons C, Catapano AL, et al. 2016 European guidelines on cardiovascular disease prevention in clinical practice: the sixth joint task force of the european society of cardiology and other societies on cardiovascular disease prevention in clinical practice (constituted by representatives of 10 societies and by invited experts)developed with the special contribution of the european association for cardiovascular prevention \& rehabilitation (EACPR). Eur Heart J. 2016:37:2315-81.

\section{Publisher's Note}

Springer Nature remains neutral with regard to jurisdictional claims in published maps and institutional affiliations.
Ready to submit your research? Choose BMC and benefit from:

- fast, convenient online submission

- thorough peer review by experienced researchers in your field

- rapid publication on acceptance

- support for research data, including large and complex data types

- gold Open Access which fosters wider collaboration and increased citations

- maximum visibility for your research: over $100 \mathrm{M}$ website views per year

At BMC, research is always in progress.

Learn more biomedcentral.com/submissions 